\title{
Influencia del capital humano en el desempeño organizacional de las instituciones de educación superior tecnológica en México
}

\author{
Felipe Machorro $^{1^{*}}$ y María V. Romero ${ }^{2}$ \\ (1) Universidad de las Américas Puebla, Ex hacienda Sta. Catarina Mártir s/n. San Andrés Cholula, Puebla, México, \\ (correo-e: felipe.machorro@udlap.mx) \\ (2) Instituto Tecnológico Superior de Tierra Blanca, Prolongación Avenida Veracruz s/n, Tierra Blanca, Veracruz, \\ México, (correo-e: vromero@itstb.edu.mx) \\ * Autor a quien debe ser dirigida la correspondencia.
}

Recibido Dic. 24, 2020; Aceptado Feb. 23, 2021; Versión final Abr. 1, 2021, Publicado Oct. 2021

\begin{abstract}
Resumen
El principal objetivo de este estudio es analizar la influencia del capital humano en el desempeño organizacional de las instituciones de educación superior en México. Algunos estudios empíricos han encontrado una relación directa, mientras que otros la han estudiado de forma indirecta mediante el capital estructural y relacional. Se realizó una investigación correlacional en la que se aplicó una encuesta sobre la percepción de capital intelectual y desempeño a 149 mandos medios y directivos. Mediante un modelo de ecuaciones estructurales por mínimos cuadrados parciales, se analizó la influencia de las dimensiones del capital humano en el desempeño. Los resultados muestran la influencia directa del desarrollo del capital humano en el desempeño organizacional, así como una influencia indirecta de la capacidad del capital humano. En conclusión, se valida la importancia del capital humano en el desempeño de las instituciones de educación superior.
\end{abstract}

Palabras clave: capital humano; capital intelectual; desempeño organizacional; educación superior; México

\section{Influence of human capital on the organizational performance of technological higher education institutions in Mexico}

\begin{abstract}
The primary aim of this research study is to examine the influence of human capital on the organizational performance of higher education institutions in Mexico. A number of previous empirical studies have reported a direct relationship, but others have reported an indirect relationship through structural and relational capital. The methodology used is correlational-based. A survey on the perception of intellectual capital and performance is applied to 149 managers and directors. The influence of the dimensions of human capital on performance is analyzed by using a partial least squares structural equation model. The results show that there is a direct influence of human capital development and an indirect influence of human capital capacity on organizational performance. In conclusion, the results validate the importance of human capital for higher education institutions' performance.
\end{abstract}

Keywords: human capital; intellectual capital; organizational performance; higher education; Mexico 


\section{INTRODUCCIÓN}

El desempeño organizacional, definido en términos sencillos como un indicador de lo bien que una organización logra sus objetivos (Li-An, 2011), es uno de los constructos de mayor interés dentro de la ciencia administrativa. Su estudio es esencial para permitir a los investigadores y gerentes valorar las acciones que las organizaciones y sus administradores realizan, dentro de un entorno cambiante que cada vez representa mayor competencia (Xianhai y Michael, 2011). Por tanto, una de las principales preocupaciones de los investigadores ha sido encontrar los factores que impulsan un mejor desempeño de las organizaciones, así como diseñar instrumentos de evaluación aplicables para dichas variables dentro de instituciones de educación superior (Machorro y Romero, 2017).

Actualmente existe cierto consenso sobre el efecto positivo del capital intelectual en las organizaciones. Sin embargo, aún no queda claro la interacción de sus componentes (se identifican típicamente tres: capital humano, capital estructural y capital relacional) y la forma en que cada uno de ellos contribuyen con el desempeño organizacional. El caso más discutido es el papel que juega el capital humano y el efecto que tiene en el desempeño: por una parte, se identifica evidencia de un efecto positivo directo (Youndt y Snell, 2020; Chen et al., 2021; Li et al., 2021). Por otra parte, autores como Bontis (1998) consideran que el efecto del capital humano en el desempeño organizacional es indirecto, mediado por el capital estructural y el capital relacional. Este modelo ha sido replicado, encontrando evidencia favorable por Mention y Bontis (2013), Bontis et al. (2018) y Asiaei et al. (2020). Otro ejemplo interesante es el encontrado en Orlandini (2020) en el que se analiza el impacto del perfil femenino en el capital humano en el desempeño organizacional, pero mediado por la orientación al mercado, es decir, con un efecto indirecto.

Esta contradicción entre los estudios previos da origen a la presente investigación, además del hecho de que todos los estudios previos han abordado la contribución al desempeño organizacional de los componentes del capital intelectual (capital humano, capital estructural y capital relacional), dejando pendiente el análisis desagregado de la influencia de las dimensiones de cada componente del capital intelectual. Por lo anterior, el objetivo de esta investigación fue estudiar la contribución de las dimensiones del capital humano en las dimensiones del desempeño organizacional en el contexto de los Institutos Tecnológicos Descentralizados del estado de Veracruz. Para ello se utilizaron las dimensiones encontradas en Machorro (2013), tanto para los componentes del capital intelectual como para el desempeño organizacional.

Para lograr el objetivo planteado, este documento se compone de las siguientes secciones: posterior a la presente introducción se plantean algunos conceptos relevantes del capital intelectual, tales como las dimensiones que lo componen (capital humano, capital estructural y capital relacional). En la siguiente sección se describe la metodología utilizada en la investigación para después presentar los resultados obtenidos, la discusión de los mismos y las conclusiones correspondientes.

\section{OTROS ANTECEDENTES}

El capital intelectual es definido como todo aquel conocimento que genera valor para la organización (Edvinsson y Malone, 2000), este conocimiento puede tener distintos orígenes: para la mayoría de los autores, los tres componentes del capital intelectual son el capital humano, el capital estructural y el capital relacional. En esta sección se revisa el concepto de cada uno de ellos y las dimensiones de los mismos. Además se presenta el modelo de medición propuesto para el desempeño organizacional en las instituciones de educación superior.

\section{Capital Humano}

El capital humano es definido como el conjunto de conocimientos, capacidades, habilidades y destrezas que poseen los individuos para resolver problemas que ponen a disposición de la organización pero que no pertenecen a ella, sino a las personas (Brooking, 1996; Edvinsson y Malone, 2000). La idea de que el capital humano tiene una influencia positiva en el desempeño organizacional se ha estudiado ampliamente (Carmeli y Tishler, 2004). De acuerdo con Bontis (1998) el capital humano es importante ya que es la fuente de innovación y renovación estratégica, por lo tanto, a medida que las personas adquieren más educación y formación son capaces de impulsar la producción de bienes y servicios, así como nuevas innovaciones en el mercado (Seleim et al., 2007).

Respecto a los componentes del capital humano, Bueno (2003), propone tres elementos: i) Valores y actitudes, en los cuales agrupa variables tales como sentimiento de pertenencia y compromiso, automotivación, satisfacción, sociabilidad, flexibilidad, adaptabilidad y creatividad; ii) Aptitudes, comprenden todo aquello que los trabajadores saben, por ejemplo, la educación formal, formación especializada, experiencia y desarrollo personal; y iii) Capacidades, lo que el trabajador sabe hacer, aprendizaje, colaboración, comunicación y liderazgo. 
Para Roos et al., (1997), el capital humano está compuesto por la competencia (conocimientos y habilidades), actitudes (por ejemplo, motivación, comportamiento y conducta) y agilidad intelectual. De manera empírica, Huang et al., (2007), determinan los componentes del capital humano, distinguiendo entre 1) capacidad de los empleados tales como el conocimiento relacionado con el trabajo, el know-how, creatividad e innovación; 2) desarrollo y retención de los empleados, que implica la capacitación, rotación, costo por reclutamiento, experiencia previa de los empleados; comportamiento de los empleados, como motivación, satisfacción, lealtad, calidad del liderazgo.

Machorro (2013) distingue dos dimensiones: 1) la capacidad del capital humano, la cual está determinada por el nivel educativo del personal, así como su experiencia, creatividad y capacidad de resolver problemas; y 2) el desarrollo del capital humano, el cual hace referencia a todos los elementos que permiten que las personas incrementen sus capacidades en beneficio de la institución, por ejemplo, la capacitación, la motivación, el soporte socio-emotivo y el liderazgo. Ambas dimensiones del capital humano fomentarán un mejor desempeño por parte de los empleados de las universidades y mejorarán los resultados obtenidos.

\section{Capital Estructural}

El conocimiento que reside en la organización y que permanece en ella aun cuando las personas la abandonen se conoce como capital estructural: recursos intangibles capaces de generar valor, que residen en la propia organización (aquel que queda en la organización cuando las personas se marchan). Es decir, el valor del conocimiento creado en la organización y que se materializa en sus sistemas y desarrollos tecnológicos (Edvinsson y Malone, 2000). Debido a que todo conocimiento organizacional es originado por las personas, el nivel de capital estructural estará determinado por el capital humano; sin embargo, al mismo tiempo representa la infraestructura que da soporte al capital humano (Shih et al., 2010); es decir, proporciona el entorno que estimula a las personas a poner a disposición de la organización su conocimiento para crear valor.

Edvinsson y Malone (2000) proponen tres componentes para el capital estructural: capital organizacional, capital de innovación y capital de proceso. Por su parte Roos et al., (1997) consideran que los componentes del capital estructural son: la organización que recoge los elementos internos actualmente operativos como la infraestructura, rutinas, procesos organizativos, bases de datos, cultura empresarial, estilos de dirección, entre otros; y renovación y desarrollo que se refiere a aquellos elementos que aludirían a la capacidad de renovación de la empresa y a los resultados de la innovación en forma de derechos comerciales protegidos, propiedad intelectual, patentes, etc. Huang y Hsueh (2007), desagregan al capital estructural en dos componentes: desarrollo de nuevos productos e ideas (implementación de nuevas ideas, efectividad del gasto en investigación y desarrollo, patentes, derechos de autor y marcas), e infraestructura de la organización, en la cual contemplan los sistemas, bases de datos, uso de tecnologías de información y la cultura organizacional.

En el contexto de las instituciones de educación superior Machorro (2013), considera tres dimensiones del capital estructural: 1) gestión del conocimiento, el cual contempla la investigación y producción científicas de las universidades, es decir, la generación del conocimiento dentro de la institución, así como los manuales y procedimientos que estructuran los procesos y los sistemas de control y evaluación; 2) tecnología de información y comunicación (TIC's), que comprenden los equipos de cómputo y aplicaciones informáticas para desarrollar las actividades académicas y administrativas; y 3) la infraestructura para llevar a cabo el proceso de enseñanza aprendizaje.

\section{Capital Relacional}

El capital relacional es el valor que tienen las relaciones existentes entre la empresa y los diferentes agentes económicos y sociales con los que interacciona en el desarrollo de su actividad, tales como clientes, proveedores, socios, accionistas, inversores etc. (Marr et al., 2003). Es decir, es el conocimiento que se encuentra incluido en las relaciones de la organización con el entorno (Bontis, 1998), incluye el valor que generan las relaciones de la empresa con todos sus grupos de interés e implica tanto las relaciones de la empresa con terceros como las percepciones que éstos tengan de la compañía (Sveiby, 1997; Edvinsson y Malone, 2000). Al ser los integrantes de la organización quienes crean y mantienen las relaciones con los agentes externos, el capital humano determina al capital relacional (Welbourne y Pardo-del-Val, 2009). Estas relaciones incrementan la capacidad de la organización para generar ventajas competitivas y así mejorar su desempeño organizacional (Kamukama et al., 2010).

Respecto a las dimensiones del capital relacional, Bontis (1998) propuso el concepto de capital cliente, el cual contempla solamente las relaciones de la empresa con sus clientes. Así, este capital representa el conocimiento presente de los canales de distribución y de las relaciones con clientes desarrolladas por la organización como resultado de su actividad. Sin embargo, definiciones posteriores ampliaron esta categoría 
denominándola capital relacional, la cual integra el conocimiento presente en todas las relaciones que una organización desarrolla, sea con clientes, competidores, proveedores, etc. (Sharabati et al. 2010).

Así, el capital relacional abarca no sólo a los clientes sino también a los demás agentes económicos y sociales que se relacionan con la organización. Sveiby (1997), establece una dimensión denominada estructura externa, la cual se refiere al valor que tiene para la empresa el conjunto de relaciones que mantiene con el exterior. En ella incluye las relaciones con los clientes y proveedores, los nombres de los productos, las marcas registradas, la reputación o imagen de la empresa. Por su parte, Brooking (1996) los denomina activos de mercado, los cuales constituyen el potencial que la empresa posee resultante de los intangibles que guardan relación con el mercado y que le otorgan una ventaja competitiva. Algunos ejemplos son las marcas, la clientela y su fidelidad, la reserva de pedidos, los canales de distribución, diversos contratos y acuerdos tales como licencias, franquicias, etc.

En el Modelo Intellectus (CIC, 2003), el capital relacional se divide en dos bloques: el capital negocio que se refiere al valor que representan las relaciones que mantiene la empresa con los principales agentes vinculados con su actividad y el capital social, el cual integra el valor que representan las relaciones que la empresa mantiene con los restantes agentes sociales que actúan en su entorno, expresado en términos del nivel de integración, compromiso, cooperación, cohesión, conexión y responsabilidad social que quiere establecer con la sociedad. En su taxonomía empírica, Huang et al. (2007) dividen al capital relacional en perspectivas de mercado (crecimiento y potencial), los datos sobre los clientes y la relación que se tiene con los consumidores (satisfacción, lealtad, quejas).

En Machorro (2013), se proponen las relaciones externas y la imagen y comunicación como dimensiones del capital relacional en Instituciones de Educación Superior. En el caso de las relaciones externas, estas miden la calidad de las relaciones con agentes del medio ambiente de la universidad, por ejemplo, las instituciones de educación media, otras universidades y organismos gubernamentales. También en esta dimensión se incluyen las actividades de vinculación, extensión y difusión de la cultura. Por su parte, la imagen y comunicación hace referencia a la manera en que la universidad tiene contacto con actores como padres de familia, egresados y empleadores, así también como la reputación y confianza que goza la institución.

\section{Desempeño organizacional}

La medición del desempeño organizacional en las instituciones de educación superior es un tema que se ha discutido ampliamente en la literatura (Xianhai y Michael, 2011), ya que derivado de las características particulares de este tipo de organizaciones se plantean algunos retos para su definición, tales como lograr un consenso respecto de los objetivos que las universidades deben lograr, los interesados en sus resultados y a quién debe rendir cuentas la institución.

En la presente investigación se tomó como base para la medición del desempeño de las universidades el modelo de Machorro (2013), el cual, a partir del modelo de excelencia administrativa de la Fundación Europea de Gestión de la Calidad (EFQM), determinó tres dimensiones: 1) satisfacción de agentes directos, la cual se enfoca en medir el nivel la satisfacción de los estudiantes, así como la medida en la cual la universidad actúa oportunamente en función de estos resultados; 2) resultados de gestión, que indican el grado en que se han logrado los objetivos de la institución, así como los resultados objetivos respecto de la gestión del personal, la gestión económica y la satisfacción de los empleados; y 3) desempeño comparado, el cual analiza los resultados obtenidos por parte de la institución en función de aquellos reportados por universidades similares.

\section{METODOLOGÍA}

La investigación es de tipo exploratoria, descriptiva y correlacional. Debido al carácter exploratorio del estudio no se han planteado hipótesis para el mismo. En su lugar se planteó un modelo en el cual se estudia la relación de las dos dimensiones del capital humano con las tres dimensiones del desempeño organizacional. En caso de que alguna de las dimensiones no presentara un efecto directo en el desempeño organizacional, se planteó un nuevo modelo en el que su explora el efecto indirecto.

\section{Participantes}

Para estudiar la relación entre los componentes del capital intelectual y el desempeño organizacional se tomaron en cuenta ocho instituciones de educación superior tecnológica en México en las cuales fue aplicado el instrumento. Dado que las variables requieren la comprensión de datos de desempeño en un nivel organizacional se han tomado en cuenta únicamente a los mandos medios y directivos. La población encuestada fueron los jefes de departamento, jefes de división, subdirectores, directores de área y directores generales de los ocho tecnológicos que accedieron a formar parte del estudio. En todos los casos, la aplicación del instrumento fue realizada de manera personal en las áreas de trabajo de los participantes, obteniéndose 147 cuestionarios válidos de ocho diferentes institutos tecnológicos. 


\section{Instrumento}

Para medir el desempeño organizacional y el capital intelectual se utilizó el instrumento desarrollado en Machorro (2013) y validado en el contexto de universidades estatales en México por Mercado, Gil-Monte, y Demuner (2014), el cual presenta las características resumidas en la Tabla 1.

Tabla 1: Dimensiones e ítems del instrumento aplicado

\begin{tabular}{|c|c|c|c|c|}
\hline Variable & Dimensión & $\begin{array}{l}\text { No. de } \\
\text { ítems }\end{array}$ & $\begin{array}{c}\text { Alfa de } \\
\text { Cronbach }\end{array}$ & Opciones de respuesta \\
\hline \multirow{3}{*}{$\begin{array}{l}\text { Desempeño } \\
\text { organizacional }\end{array}$} & Satisfacción de agentes directos & 7 & 0,927 & \multirow[t]{4}{*}{4 opciones en escala Likert } \\
\hline & Resultados de gestión & 5 & 0,944 & \\
\hline & Desempeño comparado & 6 & 0,928 & \\
\hline \multirow[t]{2}{*}{ Capital humano } & Capacidad del $\mathrm{CH}$ & 7 & 0,932 & \\
\hline & Desarrollo del $\mathrm{CH}$ & 8 & 0,951 & \multirow{6}{*}{$\begin{array}{l}10 \text { opciones en diferencial } \\
\text { semántico donde } 10 \text { : } \\
\text { adecuado y } 1 \text { : inadecuado }\end{array}$} \\
\hline \multirow[t]{3}{*}{ Capital estructural } & Gestión del conocimiento & 9 & 0,933 & \\
\hline & Infraestructura & 2 & 0,813 & \\
\hline & TICs & 2 & 0,810 & \\
\hline \multirow[t]{2}{*}{ Capital relacional } & Imagen institucional & 8 & 0,938 & \\
\hline & $\begin{array}{l}\text { Relaciones y actividades } \\
\text { externas }\end{array}$ & 7 & 0,934 & \\
\hline
\end{tabular}

\section{Análisis estadístico}

El análisis de la relación entre las variables latentes se llevó a cabo a través de un modelo de ecuaciones estructurales estimado por el método de Mínimos Cuadrados Parciales (PLS). La elección del método PLS para este estudio, se debe al carácter exploratorio de esta investigación. El uso de SEM basado en covarianzas no solo se recomienda para muestras más grandes, sino también para modelos estructurales que ya se encuentran bien consolidados en el desarrollo de sus escalas de medida. En el presente estudio, el instrumento y el modelo a probar se encuentra en desarrollo aún. La estimación se llevó a cabo con el paquete computacional SmartPLS.

\section{RESULTADOS}

La Tabla 2 muestra los resultados del modelo estructural que evalúa la influencia directa de las dimensiones de capital humano en el desempeño organizacional.

Tabla 2: Resultados modelo estructural modelo completo

\begin{tabular}{|l|c|c|}
\hline Relación & Coeficiente $\beta$ & Valor $p$ \\
\hline Capacidad de CH -> Desempeño comparado & 0,111 & 0,363 \\
\hline Capacidad de CH -> Resultados de gestión & $-0,008$ & 0,945 \\
\hline Capacidad de CH -> Satisfacción agentes directos & $-0,008$ & 0,947 \\
\hline Desarrollo de CH -> Desempeño comparado & 0,522 & 0,000 \\
\hline Desarrollo de CH -> Resultados de gestión & 0,686 & 0,000 \\
\hline Desarrollo de CH -> Satisfacción agentes directos & 0,662 & 0,000 \\
\hline
\end{tabular}

Para poder calcular los valores $p$ de los coeficientes de regresión se llevó a cabo un análisis de remuestreo con 500 submuestras. Puede apreciarse que la dimensión desarrollo de capital humano tiene una influencia positiva y significativa en las tres dimensiones de desempeño organizacional. En esta dimensión se incluyen por principio las relaciones internas entre los miembros de la organización, así como las características propias de las instituciones, tales como la capacitación, liderazgo, motivación, soporte socioeconómico; es decir, este factor corresponde a los esfuerzos de la organización por incrementar su capital humano 
El caso contrario sucede con la dimensión capacidad del capital humano ya que no presenta un nivel de significancia estadística para ninguna dimensión de desempeño organizacional. Esta dimensión representa las características que el personal aporta a la institución como parte del capital humano: aptitudes y capacidades, nivel educativo, experiencia, creatividad, etc.; es decir, considera la capacidad de los empleados para generar valor. Para esta dimensión se diseñaron dos modelos para evaluar su influencia indirecta sobre el desempeño organizacional.

El primero de estos modelos relaciona a la capacidad de capital humano con las dimensiones de capital estructural, mientras que el segundo mide la influencia indirecta sobre el desempeño organizacional con el capital relacional como variable mediadora. En la Tabla 3 se resumen los resultados del modelo estructural que relaciona a la capacidad del capital humano con el desempeño organizacional mediante las dimensiones del capital estructural. Puede apreciarse que la capacidad del capital humano es un fuerte predictor del capital estructural ya que presenta un coeficiente positivo y significativo para sus tres dimensiones. Los efectos indirectos de la capacidad del capital humano sobre los componentes del desempeño organizacional se presentan en la Tabla 4.

Tabla 3: Modelo estructural capacidad de capital humano-capital estructural

\begin{tabular}{|l|c|c|}
\hline Relación & Coeficiente $\beta$ & Valor $p$ \\
\hline Capacidad CH -> Gestión del conocimiento & 0,845 & 0,000 \\
\hline Capacidad CH -> Infraestructura & 0,606 & 0,000 \\
\hline Capacidad CH -> TICs & 0,629 & 0,000 \\
\hline Gestión del conocimiento -> Desempeño comparado & 0,464 & 0,000 \\
\hline Gestión del conocimiento -> Resultados de gestión & 0,534 & 0,000 \\
\hline Gestión del conocimiento -> Satisfacción agentes directos & 0,581 & 0,000 \\
\hline Infraestructura -> Desempeño comparado & 0,019 & 0,818 \\
\hline Infraestructura -> Resultados de gestión & 0,055 & 0,523 \\
\hline Infraestructura -> Satisfacción agentes directos & 0,012 & 0,899 \\
\hline TICs -> Desempeño comparado & 0,167 & 0,056 \\
\hline TICs -> Resultados de gestión & 0,130 & 0,132 \\
\hline TICs -> Satisfacción agentes directos & 0,079 & 0,381 \\
\hline
\end{tabular}

Tabla 4: Efectos indirectos capacidad de capital humano con mediación de capital estructural

\begin{tabular}{|l|c|c|}
\hline Relación & Coeficiente $\beta$ & Valor $p$ \\
\hline Capacidad de $\mathrm{CH}$-> Desempeño comparado & 0,508 & 0,000 \\
\hline Capacidad de $\mathrm{CH}$-> Resultados de gestión & 0,566 & 0,000 \\
\hline Capacidad de $\mathrm{CH}$-> Satisfacción agentes directos & 0,548 & 0,000 \\
\hline
\end{tabular}

De acuerdo con los resultados de la Tabla 4 se confirma la influencia indirecta de la capacidad del capital humano sobre el desempeño organizacional. Puede apreciarse que el efecto indirecto es explicado por la influencia de la capacidad del capital humano sobre la gestión del conocimiento ya que esta dimensión es la única de las tres que componen al capital estructural en tener un efecto significativo sobre el desempeño organizacional. Respecto a la influencia indirecta de la capacidad del capital humano por mediación del capital relacional, la Tabla 5 presenta los resultados del modelo estructural.

En este caso puede apreciarse un comportamiento similar que en el capital estructural: la capacidad del capital humano influye positiva y significativamente en ambas dimensiones del capital relacional; sin embargo, la única de ellas que determina al desempeño organizacional es la dimensión imagen y comunicación. El resumen de los efectos indirectos de la capacidad de capital humano en el desempeño mediante el capital relacional se muestra en la Tabla 6 . De esta forma se confirma nuevamente la influencia indirecta de la capacidad del capital humano en el desempeño organizacional. 
Tabla 5: Modelo estructural capacidad de capital humano-capital relacional

\begin{tabular}{|l|c|c|}
\hline Relación & Coeficiente $\beta$ & Valor $p$ \\
\hline Capacidad CH -> Imagen y comunicación & 0,845 & 0,000 \\
\hline Capacidad CH -> Relaciones externas & 0,606 & 0,000 \\
\hline Imagen y comunicación -> Desempeño comparado & 0,464 & 0,000 \\
\hline Imagen y comunicación -> Resultados de gestión & 0,534 & 0,000 \\
\hline Imagen y comunicación -> Satisfacción agentes directos & 0,581 & 0,000 \\
\hline Relaciones externas -> Desempeño comparado & 0,019 & 0,818 \\
\hline Relaciones externas -> Resultados de gestión & 0,055 & 0,523 \\
\hline Relaciones externas -> Satisfacción agentes directos & 0,012 & 0,899 \\
\hline
\end{tabular}

Tabla 6: Efectos indirectos capacidad de capital humano con mediación de capital estructural

\begin{tabular}{|l|c|c|}
\hline Relación & Coeficiente $\beta$ & Valor $p$ \\
\hline Capacidad de $\mathrm{CH}$-> Desempeño comparado & 0,535 & 0,000 \\
\hline Capacidad de $\mathrm{CH}$-> Resultados de gestión & 0,559 & 0,000 \\
\hline Capacidad de $\mathrm{CH}$-> Satisfacción agentes directos & 0,593 & 0,000 \\
\hline
\end{tabular}

\section{DISCUSIÓN}

El capital intelectual, como un activo intangible, debe crear valor a las organizaciones. Como tal, es frecuente escuchar la afirmación de que el capital humano es el recurso más valioso en las empresas. Este documento tuvo por objetivo verificar el mecanismo por el cual el capital humano es capaz de generar valor en las Instituciones de Educación Superior en México.

Bajo esta premisa se exploraron diferentes modelos estructurales para definir la naturaleza y grado de influencia de las dos dimensiones detectadas: capacidad y desarrollo de capital humano. Al respecto, de acuerdo con los resultados empíricos obtenidos, puede inferirse que la dimensión de desarrollo humano tiene un efecto directo y significativo en las tres dimensiones de desempeño organizacional que se evaluaron. Esta dimensión hace referencia a las acciones que la institución realiza para incrementar su nivel de capital humano: capacitación, liderazgo, motivación, soporte socioeconómico, lo cual brinda evidencia de la importancia de tales procesos ya que, como puede apreciarse, generan efectos directos sobre el desempeño de las organizaciones.

Por el contrario, en el caso de la dimensión capacidad del capital humano, no se encontró un efecto directo sobre ninguna de las dimensiones del desempeño organizacional. Esta capacidad incluye las características que el personal aporta a la institución: aptitudes y capacidades, nivel educativo, experiencia y creatividad. Sin embargo, estas características del capital humano sí tienen un efecto indirecto en las dimensiones del desempeño organizacional de las universidades al determinar los componentes del capital estructural y el capital relacional de las mismas. Estos resultados concuerdan con las hipótesis planteadas en el modelo de Bontis (1998), respecto a que la influencia del capital humano en el desempeño es de carácter indirecto, tal como también se había encontrado en los estudios de Mention y Bontis (2013), Bontis et al. (2018) y Asiaei et al. (2020).

\section{CONCLUSIONES}

Los resultados de los modelos diseñados para evaluar la influencia indirecta de la capacidad del capital humano en el desempeño organizacional permiten establecer conclusiones interesantes. Por principio, se logró verificar que la capacidad de capital humano tiene influencia en todas las dimensiones tanto del capital estructural como del capital relacional. Sin embargo, no todas éstas influyen directamente en el desempeño organizacional. Es decir, la influencia indirecta del capital humano está focalizada: para el caso del capital estructural es mediante la gestión del conocimiento que la capacidad del personal puede influir sobre el desempeño; mientras que para el capital relacional es la imagen y comunicación de la institución la que opera como variable mediadora. Las limitaciones del estudio tienen que ver con el diseño de muestreo, ya que la muestra utilizada fue por conveniencia, dado que se encuestó únicamente a los directivos de las instituciones que accedieron a ello, pudiendo provocar un sesgo en los resultados. Además, las variables fueron medidas mandos medios y directivos de los institutos tecnológicos, sin tomar la opinión de los agentes externos, tales como los alumnos, egresados, padres de familia y empleadores, actores importantes en la percepción del desempeño de las instituciones de educación superior. 


\section{REFERENCIAS}

Asiaei, K., Barani, O., Bontis, N., y Arabahmadi, M., Unpacking the black box. How intrapreneurship intervenes in the intellectual capital-performance relationship?, Journal of Intellectual Capital, 21(6), 809-834 (2020)

Bontis, N., Intellectual capital: an exploratory study that develops measures and models, Management Decision, 36(2), 63-76 (1998)

Bontis, N., Ciambotti, M., Palazzi, F., y Sgro, F., Intellectual capital and financial performance in social cooperative enterprises, Journal of Intellectual Capital, 19(4), 712-731 (2018)

Brooking, A., El capital intelectual. El principal activo de las empresas del tercer milenio, Ediciones Paidós Ibérica S.A., Barcelona, España (1996)

Bueno, E., Modelo Intellectus: medición y gestión del capital intelectual, DICREA creaciones gráficas, Madrid, España (2003)

Carmeli, A., y Tishler, A., The relationships between intangible organizational elements and organizational performance, Strategic Management Journal, 25(13), 1257-1278 (2004)

Chen, M. Y., Lam, L. W., y Zhu, J. N., Should companies invest in human resource development practices? The role of intellectual capital and organizational performance improvements, Personnel Review, 50(2), 460-477 (2021)

CIC, Modelo Intellectus: medición y gestión del capital intelectual, Centro de Investigación sobre la Sociedad del Conocimiento, Madrid, España (2003)

Edvinsson, L., y Malone, M., El capital intelectual: como identificar y calcular el valor de los recursos intangibles de su empresa, Ediciones Gestión, Barcelona, España (2000)

Huang, C. C., Luther, R., y Tayles, M., An evidence-based taxonomy of intellectual capital, Journal of Intellectual Capital, 8(3), 386-408 (2007)

Huang, C. F., y Hsueh, S. L., A study on the relationship between intellectual capital and business performance in the engineering consulting industry: a path analysis, Journal of Civil Engineering and Management, 13(4), 265-271 (2007)

Kamukama, N., Ahiauzu, A., y Ntayi, J. M., Intellectual capital and performance: testing interaction effects, Journal of Intellectual Capital, 11(4), 554-574 (2010)

$\mathrm{Li}, \mathrm{Y}$., Gong, Y., y otros 5 autores, Leveraging age diversity for organizational performance: an intellectual capital perspective, Journal of Applied Psychology, 106(1), 71-91 (2021)

Li-An, H., Meditation, learning, organizational innovation and performance, Industrial Management \& Data Systems, 111(1), 113-131 (2011)

Machorro, F., Influencia del capital intelectual en el desempeño organizacional de los institutos tecnológicos descentralizados del estado de Veracruz, 2012, Universidad Autónoma del Estado de México (2013)

Machorro, F., y Romero, M. V., Propuesta de un instrumento de autoevaluación del desempeño organizacional en instituciones públicas de educación superior en México, Formación Universitaria, 10(3), 3-10 (2017)

Marr, B., Gray, D., y Neely, A., Why do firms measure their intellectual capital?, Journal of Intellectual Capital, 4(4), 441-464 (2003)

Mention, A., y Bontis, N., Intellectual capital and performance within the banking sector of Luxembourg and Belgium, Journal of Intellectual Capital, 14(2), 286-309 (2013)

Mercado, P., Gil-Monte, P. R., y Demuner, M. R., Development and initial validation of a survey for intellectual capital in

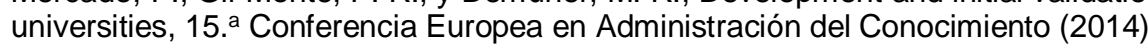

Orlandini, I. E., El perfil directivo femenino y su relación con la orientación al mercado y el desempeño organizacional, Información Tecnológica, 31(3), 241-248 (2020)

Roos, J., Roos, G., Dragonetti, N. C., y Edvinsson, L., Capital intelectual. El valor intangible de la empresa, Paidós, Barcelona, España (1997)

Seleim, A., Ashour, A., y Bontis, N., Human capital and organizational performance: a study of egyptian software companies, Management Decision, 45(4), 789-801 (2007)

Sharabati, A. A., Naji, S., y Bontis, N., Intellectual capital and business performance in the pharmaceutical sector of Jordan, Management Decision, 48(1), 105-131 (2010)

Shih, K. H., Chang, C. J., y Lin, B., Assessing knowledge creation and intellectual capital in banking industry, Journal of Intellectual Capital, 11(1), 74-89 (2010)

Sveiby, K. E., The new organizational wealth: managing and measuring knowledge-based assets,. Berrett-Koehler Publishers, San Francisco, Estados Unidos (1997)

Welbourne, T. M., y Pardo-del-Val, M., Relational capital: strategic advantage for small and medium-size enterprises (SMEs) through negotiation and collaboration, Group Decision and Negotiation, 18(5), 483-497 (2009)

Xianhai, M., y Michael, M., Performance measurement models in facility management: a comparative study, Facilities, 29(11), 472-484 (2011)

Youndt, M. A., y Snell, S. A. Human resource configurations, intellectual capital, and organizational performance, Journal of Managerial Issues, 32(1), 60-83 (2020) 